\title{
Tratamiento del tabaquismo en pacientes con enfermedad pulmonar obstructiva crónica
}

\author{
SERGIO BELLO S.*, HAYDEE CHAMORRO R.**** y ANDREA BARRIENTOS R.***
}

Smoking cessation in patients with chronic obstructive pulmonary disease

In COPD patients, smoking worsens lung function and smoking cessation reduces the fall in FEV and all-cause of mortality. There is a high percentage of these patients who continues smoking despite the disease due to the high degree of dependence, low motivation to quit and association with depression and false beliefs, which hampers attempts to stop smoking and reduce their success. The smoker's treatment has two pillars: psychosocial intervention and pharmacological treatment. In COPD patients' psychosocial intervention should be especially intense, empathic and aimed at increasing motivation and self-efficacy, using Motivational Interviewing. In pharmacotherapy, first-line drugs are nicotine replacement (NRT), bupropion and varenicline. In patients with COPD, NRT is effective and may be combined passive delivery NRT with other active delivery. Bupropion has been used, with cost-effectiveness. Varenicline in patients with mild to moderate COPD has been effective. In brief therapies for smokers with COPD should be more intense, combining pharmacological and psychosocial treatment and maintained over time.

Key words: COPD, tobacco use, smoking cessation.

\section{Resumen}

En pacientes con EPOC, el consumo de tabaco empeora la función pulmonar y al dejar de fumar disminuye la caída del $\mathrm{VEF}_{1}$ y la mortalidad por todas las causas. No obstante, hay un alto porcentaje que continúa fumando a pesar de la enfermedad debido al alto grado de dependencia, baja motivación para dejar de fumar y asociación con cuadros depresivos y creencias falsas, lo que dificulta los intentos por dejar de fumar y disminuye el éxito de ellos. El tratamiento de los fumadores se basa en dos pilares: intervención psico-social y tratamiento farmacológico. En pacientes con EPOC la intervención psicosocial debe ser especialmente intensa, empática y orientada a incrementar la motivación y la autoeficacia, utilizándose la Entrevista Motivacional. En farmacoterapia, los medicamentos de primera línea son los sustitutos de nicotina (TSN), bupropión y vareniclina. En los pacientes EPOC la TSN es efectiva, pudiendo combinarse TSN de entrega pasiva con otro de entrega activa. El bupropión ha demostrado una buena relación costo-efectividad y la vareniclina, en pacientes con EPOC leve a moderada, ha sido efectiva. En resumen, las terapias para fumadores con EPOC deben ser más intensas, combinando tratamiento farmacológico y psicosocial y mantenidas en el tiempo.

Palabras clave: EPOC, consumo de tabaco, tratamiento del tabaquismo.

\section{Introducción}

El tabaquismo es la principal causa prevenible de muerte en todo el mundo. La mitad de las personas que consumen tabaco morirá a consecuencias de algunas de las muchas enfermedades que produce dicho consumo. Dentro del amplio espectro de enfermedades relacionadas con el tabaco, se encuentra la Enfermedad Pulmonar Obstructiva Crónica (EPOC) cuya causa se debe en el 80 a $90 \%$ de los casos, al tabaquismo.

La EPOC es una patología muy prevalente en el país y por ello fue incorporada dentro de las enfermedades con garantías explícitas de salud (GES). En Latinoamérica (LA) de acuerdo a datos del estudio PLATINO ${ }^{1}$, Santiago de Chile

* Programa de Control del Tabaquismo. Instituto Nacional del Tórax (INT). Santiago, Chile.

** Psicóloga.

*** Médico residente de Enfermedades Respiratorias, INT. 
presenta una alta prevalencia de EPOC lo que es concordante con su elevada prevalencia de fumadores. Este estudio fue realizado en 5 ciudades de LA: Ciudad de México, Caracas, Sao Paulo, Montevideo y Santiago y mostró una alta prevalencia de tabaquismo en LA, comparándola con Europa y EE.UU. Cabe destacar que dentro de las ciudades incluidas en el estudio, Santiago ocupa el primer lugar en cuanto a prevalencia de tabaquismo con 38,5\%, seguido de Caracas con 28,5\%, Montevideo 28\%, Ciudad de México $25,4 \%$ y Sao Paulo con 23,9\%. Por otra parte, $\mathrm{PLATINO}^{2}$ también muestra que en el grupo de los fumadores se observa una mayor prevalencia de EPOC comparado con los no fumadores. Por ejemplo en Montevideo, en el grupo de fumadores se observó $22,5 \%$ de prevalencia de EPOC vs $15,3 \%$ en los no fumadores, $21,8 \%$ vs $12,5 \%$ en Sao Paulo, $18,7 \%$ vs $15,9 \%$ en Santiago, respectivamente. Además se encontró que a mayor consumo de tabaco (expresado en paquetes/año) hay mayor prevalencia de EPOC, especialmente cuando el consumo es mayor a 20 paquetes/año, con prevalencias tan altas como 31 y $32 \%$ en Santiago y Montevideo respectivamente.

Por otra parte, dejar de fumar es la estrategia más efectiva para enlentecer o detener la progresión de la enfermedad y sigue siendo la mejor intervención para los pacientes con EPOC, a pesar de los avances en farmacoterapia de los últimos años ${ }^{3}$.

A continuación se analizará la relación tabaco y EPOC, especialmente las consecuencias de que los pacientes ya diagnosticados de esta enfermedad continúen fumando, y luego se revisará en forma más detallada el tratamiento actual de los fumadores con EPOC.

\section{Relación EPOC y tabaco}

De acuerdo a GOLD Update $2011^{4}$, el tratamiento de los enfermos con EPOC debe tener como grandes objetivos reducir los síntomas (especialmente mejorar la tolerancia al ejercicio) y por otra parte, reducir los riesgos futuros (prevenir la progresión de la enfermedad, prevenir y tratar las exacerbaciones y reducir la mortalidad). En este contexto dejar de fumar se convierte en la intervención clave en aquellos pacientes con EPOC que continúan fumando, ya que esta conducta impediría el cumplimiento de dichos objetivos.

De acuerdo a recientes ensayos clínicos a gran escala ${ }^{5,6}$ un porcentaje muy alto, que fluctúa entre 30 a 43\%, de los pacientes con EPOC moderada a severa, continúan fumando, no obstante su enfermedad.
Si un fumador con EPOC establecida continúa fumando se produce un empeoramiento progresivo de la función pulmonar: De acuerdo a datos del Lung Health Study (LHS) ${ }^{7}$, estudio de casi 4.000 pacientes con EPOC seguidos por 5 años, quienes persisten fumando perdieron $62 \mathrm{ml} / \mathrm{año}$ del $\mathrm{VEF}_{1}$ en comparación con $31 \mathrm{ml} / \mathrm{año}$ entre quienes dejaron de fumar. Más recientemente, en el estudio UPLIFT, Tashkin y cols ${ }^{8}$, mostraron cifras muy similares: la caída anual del $\mathrm{VEF}_{1}$ en los pacientes con EPOC que continuaron fumando fue de $51 \mathrm{ml} /$ año y en quienes dejaron de hacerlo fue de $23 \mathrm{ml} / \mathrm{año}$. Dicho de otra manera, dejar de fumar cambia la historia natural de la EPOC, corroborando los datos de Fletcher y Peto de la década del 70.

Se ha reportado también que si un paciente con EPOC continúa fumando presenta un aumento de la frecuencia de exacerbaciones, además del efecto deletéreo sobre la función pulmonar, de acuerdo a otra publicación del LHS $^{9}$, con una caída adicional del $\mathrm{VEF}_{1}$ de $7 \mathrm{ml} / \mathrm{año}$ en los fumadores con promedio de 1 exacerbación anual en los 5 años de seguimiento del estudio.

Por otra parte, y de acuerdo a datos del LHS, la mortalidad, ya sea por enfermedades cardiovasculares, cáncer pulmonar y otras enfermedades respiratorias disminuyen en quienes dejan de fumar, en el seguimiento a 14,5 años ${ }^{10}$. Todas las causas de mortalidad fueron significativamente menores en los que dejaron de fumar totalmente (6,0 por 1.000 pacientes/año) en comparación con quienes dejaron en forma intermitente y los que continuaron fumado $(7,7 \mathrm{y} 11,0$ por 1.000 pacientes/año), respectivamente.

\section{¿Por qué los fumadores con EPOC siguen fumando?}

Esta es una pregunta primordial para comprender el hecho de que estos pacientes, a pesar de saber que su enfermedad está directamente relacionada con su consumo de tabaco, persisten en el hábito de fumar. De este conocimiento pueden derivar posibles intervenciones que contribuyan a apoyar mejor a estos pacientes en sus intentos de dejar de fumar.

Una de las causas más relevantes para explicar la persistencia en el consumo de tabaco es la condición de alto grado de dependencia a la nicotina. De acuerdo a un estudio de Jiménez Ruiz en España ${ }^{11}$, los fumadores con EPOC tienen más alto puntaje en la escala de Fagerström $(4,7$ vs 3,1) y fuman más cigarrillos/día $(24,2$ vs $18,3)$ que los fumadores sin EPOC. Además el 
$30 \%$ de los fumadores con EPOC tienen 7 ó más puntos en la escala de Fagerström comparado con el 10\% de los que no tienen EPOC. Es importante señalar que por cada punto adicional en la escala de Fagerström hay un aumento de $11 \%$ en la probabilidad de desarrollar EPOC ${ }^{12}$.

Los fumadores con EPOC tienen una baja motivación para dejar de fumar. De acuerdo al estudio de Jiménez Ruiz ${ }^{11}$ casi el $50 \%$ de los pacientes con EPOC estaba en fase de pre contemplación, el 35\% nunca había realizado un intento serio por dejar el tabaco y sólo el $9 \%$ de los fumadores con EPOC estaba en etapa de preparación para dejar de fumar, que es la situación en que el paciente está proclive para pasar a la "acción" de dejar de fumar.

Otra característica de los fumadores con EPOC es la frecuente asociación con cuadros depresivos. Se han publicado cifras variables pero siempre elevadas, que fluctúan entre $25 \mathrm{y}$ $50 \%$ de prevalencia de esta enfermedad en los pacientes con $\mathrm{EPOC}^{13,14}$. En pacientes con EPOC severa se encontraron prevalencias de depresión incluso mayores (entre 37 y $71 \%$ ) comparables o superiores a las observadas en enfermedades como cáncer, SIDA, enfermedad cardíaca o renal avanzadas ${ }^{15}$.

Los pacientes con EPOC presentan un amplio espectro de síntomas que oscilan desde síntomas depresivos de corta duración hasta la distimia crónica o depresión clínica, determinando una peor calidad de vida y menor adherencia al tratamiento de su enfermedad de base. Hay también evidencia acerca de una significativa sobreposición entre los síntomas de depresión y la presentación clínica de la EPOC, lo que se asocia con subdiagnóstico de la depresión, puesto que ambas entidades clínicas se asocian con fatiga aumentada, alteraciones del sueño y del apetito, dificultades de concentración y lentificación. Los pacientes depresivos con EPOC tienen más dificultad en el aprendizaje e implementación de los planes de tratamiento y en el curso de una exacerbación emergente podrían ser más lentos para solicitar ayuda médica ${ }^{14}$.

Aunque existen estudios que demuestran que estos pacientes responden satisfactoriamente a terapias farmacológicas y no farmacológicas, es preocupante la escasa cobertura de quienes reciben tratamiento efectivo ${ }^{16}$. Adicionalmente se sabe que en pacientes con depresión mayor se debería realizar primero el tratamiento de su cuadro afectivo y luego, en forma secuencial, el tratamiento del tabaquismo, lo que permitiría mayor adherencia al tratamiento y mejoría de las tasas de cese del tabaquismo ${ }^{17}$.
Hay una serie de creencias muy frecuentes entre los fumadores con EPOC y que favorecen la mantención del consumo. En un estudio cualitativo, Wilson et $\mathrm{al}^{18}$, encontraron creencias y prejuicios como "es muy tarde para dejar de fumar", "no encuentro la ayuda adecuada", además de "falta de motivación" para dejar de fumar. Los fumadores con EPOC ven también al cigarrillo como "un amigo", "perdiendo más si lo dejan". Además el no haber conseguido dejarlo antes lo viven como "fracaso y debilidad" en vez de aprendizaje para futuros intentos.

En resumen, los fumadores con EPOC consumen mayor número de cigarrillos al día, tienen un más alto grado de dependencia fisica, tienen más baja motivación para dejar de fumar, pueden estar deprimidos y sostener creencias falsas respecto del fumar, todo lo cual favorece la mantención del consumo, dificulta los intentos por dejar de fumar y reduce las probabilidades de éxito cuando se lo proponen.

\section{Tratamiento de fumadores con EPOC}

Si bien existen muchas guías de tratamiento del tabaquismo, la información sobre terapia específica para los enfermos de EPOC suele ser escasa. Las guías internacionales del manejo de la EPOC también proveen poca información sobre el manejo del tabaquismo, no obstante observarse una mayor tendencia a incluir esta información en los últimos años. En todas las guías disponibles hay consenso que el enfoque debe ser similar al utilizado en los fumadores en general, con algunas particularidades especiales que se desarrollarán a continuación.

De acuerdo a las evidencias actuales el tratamiento del tabaquismo se sustenta en dos pilares, siendo ambas intervenciones necesarias ${ }^{19,20}$ para lograr los mejores resultados terapéuticos: la intervención psicosocial y el tratamiento farmacológico.

\section{Intervención psico-social}

En los pacientes con EPOC esta intervención debe ser especialmente intensa ${ }^{21}$, empática $\mathrm{y}$ orientada a incrementar la motivación, la autoeficacia y la auto-estima. Para esto se ha utilizado la estrategia denominada "Entrevista Motivacional" (EM).

La motivación para el cambio es un elemento clave para el éxito terapéutico, sobre todo en el ámbito de las conductas adictivas, donde es frecuente el abandono del tratamiento y el bajo cumplimiento de las prescripciones terapéuticas. La Entrevista Motivacional, desarrollada por 
Miller y Rollnick ${ }^{22}$, resulta especialmente útil con las personas que son reticentes a cambiar y que se muestran ambivalentes ante el cambio, ya que trabaja activando la propia motivación de las personas para el cambio y la adherencia al tratamiento. Intenta ayudar a resolver el fenómeno de la ambivalencia a través de cuatro principios básicos: expresar empatía, crear discrepancias, dar un giro a la resistencia y fomentar y reforzar la autoeficacia ${ }^{23}$.

Con la EM puede aumentarse progresivamente la disposición al cambio adquiriendo conciencia del problema y desarrollando las estrategias necesarias para superarlo. Dado su carácter de intervención centrada en el fumador, la EM intenta ayudarle a desarrollar sus propias habilidades, con vista a estimular el cambio de conducta a través de la automotivación, ayudando a los pacientes a explorar y resolver su ambivalencia respecto al consumo de tabaco. La responsabilidad para el cambio se deja en manos del individuo, utilizando estrategias más bien persuasivas que coercitivas, más de apoyo que de discusión. El objetivo global consiste en aumentar la motivación intrínseca del fumador, de manera que el cambio surja desde la persona, más que se imponga desde afuera ${ }^{24}$.

Hay evidencias que la EM es efectiva para incrementar los intentos por dejar de fumar en el futuro ${ }^{19}$ y se ha utilizado en diferentes poblaciones de fumadores ${ }^{23}$, con resultados variables. Una reciente revisión sistemática de la colaboración Cochrane ${ }^{25}$ reconoce, sin embargo, su utilidad. Aunque no hay publicaciones específicas acerca de la utilidad de la EM en pacientes con EPOC, en quienes es frecuente la falta de motivación, este recurso podría ser útil por analogía.

\section{Tratamiento farmacológico del tabaquismo en pacientes con EPOC}

En el tratamiento de los fumadores en general, los medicamentos aprobados por la Food and Drug Administration de los EE.UU. son: la Terapia de Sustitución de Nicotina (TSN) en sus 5 formas de administración (chicles, parches, spray nasal, inhalador bucal y losenges), Bupropión y Vareniclina. Existen varias otras alternativas farmacológicas (entre otros Nortriptilina y Clonidina) que se consideran de segunda línea.

\section{Terapia de sustitución de nicotina}

Esta terapia ha demostrado su efectividad en fumadores en múltiples estudios. Un metaanálisis de la colaboración Cochrane ${ }^{26}$ revisó 123 ensayos aleatorizados y encontró que todas las formas de TSN son efectivas, reportando Odds Ratio (OR) resumen de 1,77 (IC 95\% 1,66-1,88). En los pacientes con EPOC en el Lung Health Study, Anthonisen et $\mathrm{al}^{27}$, utilizaron tratamiento conductual intensivo más chicles de nicotina, con resultados favorables $(22 \%$ de abstinencia a los 5 años $v s 5 \%$ en grupo control) y sin efectos adversos serios.

Más recientemente Tönnesen et $\mathrm{al}^{28}$, utilizaron TSN sublingual en 370 pacientes portadores de EPOC, concluyendo que la TSN fue más efectiva que placebo con un OR de 1,97 (CI 95 1,06$3,67)$. Una revisión de Wagena et $\mathrm{al}^{29}$, concluye que los chicles de nicotina son una terapia útil en fumadores con EPOC y que combinados con terapia conductual, mejorarían los resultados del tratamiento.

Es posible asociar diferentes formas de TSN, por ejemplo en modalidad de un medicamento de entrega pasiva (parches), más otro producto de entrega activa (chicles) que permita la autoadministración en los momentos de urgencia por fumar. Esto permite potenciar el efecto de cada una de las formas de administración por separado y en pacientes con EPOC este tipo de asociación podría favorecer mejores resultados ${ }^{30,31}$.

Otra estrategia disponible es la Reducción del consumo, utilizando TSN, como paso previo a la cesación, indicada en fumadores no motivados en la actualidad o que se sienten incapaces de alcanzar la cesación completa. En esta estrategia se estimula al fumador a disminuir el consumo a la mitad (50\%), utilizando chicles o inhalador nasal, dentro de las primeras dos semanas y luego se intenta bajar más aún, con la meta de llegar a la abstinencia completa en un máximo de 6 meses. En una publicación reciente ${ }^{32}$, con la reducción progresiva previa a cesación se encontró que el $57 \%$ de los pacientes abandonó el consumo después de 4 meses de reducción y el $39 \%$ se mantuvo abstinente hasta el final del seguimiento, que fue de 10 meses. Como los pacientes fumadores con EPOC con frecuencia están renuentes a la cesación inmediata, esta sería una estrategia recomendable.

\section{Bupropión}

La eficacia de bupropión para dejar de fumar en fumadores en general, está claramente demostrada. Un meta-análisis, que incluyó 12 ensayos clínicos randomizados, demostró un OR de 1,56 (IC95\% 1,1-2,21) a los 12 meses en comparación con placebo ${ }^{33}$.

Tashkin et $\mathrm{al}^{34}$, en un grupo de pacientes con EPOC leve a moderada, evaluó el uso de 
bupropión versus placebo, recibiendo todos los participantes de base, consejería individual. A los 6 meses se logró abstinencia continua en el 16\% del grupo tratado con bupropión versus un $9 \%$ en el grupo con placebo $(p<0,05)$, concluyendo que bupropión es efectivo y bien tolerado.

Van Schayck et $\mathrm{al}^{35}$, trataron 225 pacientes fumadores con EPOC comparando bupropion con nortriptilina: ambos antidepresivos fueron igual de efectivos, pero bupropión fue más costoefectivo.

\section{Vareniclina}

Vareniclina es un agonista parcial de los receptores nicotínicos de acetilcolina $\alpha 4 \beta 2$, aprobado por la FDA para el tratamiento del tabaquismo. Está disponible en Chile desde el año 2007 y se ha convertido en una alternativa atractiva, no sólo por su particular mecanismo de acción, sino también por su eficacia y buena tolerancia reportada en varios estudios ${ }^{36,37}$.

En el período de post-comercialización del uso de vareniclina surgieron algunos reportes sobre posible asociación entre su uso y el riesgo de suicidio, que llevó a que las agencias reguladoras de medicamentos de EE.UU. (FDA) y británica (MHRA) determinaran en el año 2009, la obligación de incluir una advertencia sobre el posible riesgo en el prospecto del medicamento. Sin embargo, no se ha establecido la relación causal, ya que es necesario separar los posibles efectos de la vareniclina de aquellos relacionados a la cesación del tabaquismo. En una publicación reciente $^{38}$ no se reportaron efectos neuropsiquiátricos superiores a placebo, salvo alteraciones del sueño. En otro estudio a gran escala en Inglaterra $^{39}$ en una cohorte retrospectiva en más de 80.000 fumadores, no hubo clara evidencia de que el uso de vareniclina aumentara el riesgo de depresión, suicidio o pensamientos suicidas, comparado con el uso de bupropión o terapia de reemplazo de nicotina.

En junio de 2011, fue publicado un metaanálisis sobre los riesgos de efectos adversos cardiovasculares de vareniclina ${ }^{40}$, donde se concluye que su uso se asoció a un aumento significativo de efectos adversos cardiovasculares (isquemia, arritmias, insuficiencia cardíaca congestiva y muerte súbita) comparada con placebo: $1,06 \%$ vs $0,82 \%$. La FDA emitió un comunicado en que advierte que hay un aumento de los riesgos cardiovasculares con vareniclina, y que el riesgo absoluto de eventos cardiovasculares adversos en relación a su eficacia es pequeño. Más recientemente, se publicó un nuevo meta análisis ${ }^{41}$ que demuestra que el uso de vareniclina se asocia a muy bajo riesgo de efectos adversos severos cardiovasculares $(0,67 \%)$.

En fumadores con EPOC existe una publicación reciente sobre la efectividad de vareniclina. Tashkin et $\mathrm{al}^{42}$, en un grupo de 504 pacientes fumadores, que fumaban en promedio 28 cigarrillos por día, con EPOC leve a moderada, demostró la eficacia de vareniclina en la evaluación a 1 año, con OR de 4,04 (CI 2,13-7,67), comparada con placebo. En cuanto a efectos secundarios, los más frecuentes fueron náuseas, sueños vívidos e insomnio, similares a los encontrados en publicaciones previas.

\section{Comentarios finales}

Dejar de fumar completamente sigue siendo la estrategia más efectiva para preservar la función pulmonar de los pacientes con EPOC. Por lo tanto, el apoyo a los fumadores con EPOC para que dejen el consumo de tabaco es crucial para el presente y pronóstico de dichos pacientes.

En consideración a lo expuesto y considerando las principales revisiones del tema en los últimos años ${ }^{21,43,44,45}$, es posible afirmar en cuanto a las estrategias terapéuticas más adecuadas para tratar a los fumadores con EPOC lo siguiente: éstas deben ser intensas (en cuanto al tiempo de dedicación); deben combinar tratamientos farmacológicos (vareniclina, bupropión y TSN) y abordajes psico-sociales y deben ser mantenidas en el tiempo y adaptadas individualmente.

Es un imperativo técnico y ético considerar que todo el equipo de salud responsable de atender a los pacientes con EPOC participe en el esfuerzo de motivarlos y ayudarlos a dejar de fumar. Hay actualmente más y mejores herramientas de probada eficacia orientadas a lograr las metas de cesación del consumo de tabaco.

\section{Bibliografía}

1.- MENEZES A M, LÓPEZ M V, HALLAL P, MUIÑO A, PÉREZ-PADILLA R, JARDIM J, VALDIVIA G, PERTUZÉ J, MONTES DE OCA M, TÁLAMO C, VICTORA C AND THE PLATINO TEAM. Prevalence of smoking and incidence of initiation in the Latin American adult population: the PLATINO study. BMC Public Health 2009, 9:151 doi:10.1186/1471-2458-9151.

2.- MENEZES A M, PÉREZ-PADILLA R, JARDIM J, MUIÑO A, LÓPEZ M V, VALDIVIA G, MONTES DE OCA M, TALAMO C, HALLAL P, VICTORA C, FOR THE PLATINO TEAM. Chronic obstructive pulmonary 
disease in five Latin American cities (the PLATINO study): a prevalence study. Lancet 2005; 366: 1875-81.

3.- CHIANG CHEN-YUAN C. After UPLIFT: smoking cessation remains the best intervention in smokers with COPD. Int J Tuberc Lung Dis 2008; 13: 151-2.

4.- GLOBAL INITIATIVE FOR CHRONIC OBSTRUCTIVE LUNG DISEASE [GOLD]. Global Strategy for the Diagnosis, Management, and Prevention of Chronic Obstructive Pulmonary Disease, 2011. http://www.goldcopd.com/ (Consultado el 20 de julio de 2012).

5.- CALVERLEY P M, ANDERSON J A, CELli B, FERGUSON G T, JENKINS C, JONES P W, et al. Salmeterol and fluticasone propionate and survival in chronic obstructive pulmonary disease. N Engl J Med 2007; 356: 775-89.

6.- TASHKIN D, CELLI B, SENN S, BURKHART, D, KESTEN S, MENJOGE S, et al, for the UPLIFT Study Investigators. A 4-Year Trial of Tiotropium in Chronic Obstructive Pulmonary Disease. N Engl J Med 2008; 359: 1543-54.

7.- SCANLON P D, CONNETT J E, WALLER L A, ALTOSE M D, BAILEY W C, BUIST A S. Smoking cessation and lung function in mild-to-moderate chronic obstructive pulmonary disease. The Lung Health Study. Am J Respir Crit Care Med 2000; 161: 381-90.

8.- TASHKIN DP, CELLI B, KESTEN S, LYSTIG T, MEHRA S, DECRAMER M, et al. Long-term efficacy of tiotropium in relation to smoking status in the UPLIFT trial. Eur Respir J 2010; 35: 287-94.

9.- KANNER R E, ANTHONISEN N R, CONNETT J E. Lower respiratory illnesses promote $\mathrm{FEV}_{1}$ decline in current smokers but not ex smokers with mild chronic obstructive pulmonary disease: results from the lung health study. Am J Respir Crit Care Med 2001; 164: 358-64.

10.- ANTHONISEN N R, SKEANS M A, WISE R A, MANFREDA J, KANNER R E, CONNETT J E, et al. The effects of a smoking cessation intervention on 14.5-year mortality. Ann Intern Med 2005; 142: 233-9.

11.- JIMÉNEZ-RUIZ C A, MASA F, MIRAVITLLES M, GABRIEL R, VIEJO J L, VILLASANTE C, et al. Smoking characteristics: differences in attitudes and dependence between healthy smokers and smokers with COPD. Chest 2001; 119: 1365-70.

12.- JIMÉNEZ-RUIZ C A, MIRAVITLLES M, SOBRADILLO V, GABRIEL R, VIEJO J L, MASA J F, et al. Can cumulative tobacco consumption, FTND score, and carbon monoxide concentration in expired air be predictors of chronic obstructive pulmonary disease? Nicotine \& Tobacco Research 2004; 6: 649-53.

13.- WILSON I. Depression in the patient with COPD. Int J COPD 2006; 1: 61-4.

14.- NORWOOD R. Prevalence and impact of depression in chronic obstructive pulmonary disease patients. Current Opinion in Pulmonary Medicine 2006; 12: 113-7.

15.- SOLANO J P, GOMES B, HIGGINSON I J. A compa- rison of symptom prevalence in far advanced cancer, AIDS, heart disease, chronic obstructive pulmonary disease and renal disease. J Pain Symptom Manage 2006; 31: 58-69.

16.- MAURER J, REBBAPRAGADA V, BORSON S, GOLDSTEIN R, KUNIK M, YOHANNES AM, et al. Anxiety and Depression in COPD: Current Understanding, Unanswered Questions, and Research Needs. Chest 2008; 134; 43S-56S.

17.- HISADO DÍAZ M. Tratamiento del Tabaquismo en pacientes con patología depresiva. Prev Tab 2011; 13 : 160-6.

18.- WILSON J S, ELBORN J S, FITZSIMONS D. 'It's not worth stopping now': why do smokers with chronic obstructive pulmonary disease continue to smoke? A qualitative study. J Clin Nurs 2011; 20: 819-27.

19.- FIORE M C, JAÉN C R, BAKER T B, BAILEY W C, BENOWITZ N L, CURRY S J, et al. Treating Tobacco Use and Dependence: 2008 Update. Clinical Practice Guideline. Rockville, MD: US Department of Health and Human Services, Public Health Service; 2008.

20.- BELLO S, FLORES A, BELLO M, CHAMORRO H. Diagnóstico y tratamiento psicosocial del tabaquismo. Rev Chil Enf Respir 2009; 25: 218-30.

21.- DE GRANDA ORIVE J I, JAREÑO J, ROIG F. Tratamiento del tabaquismo en el EPOC: Revisión actualizada. Prev Tab 2011; 13: 117-21.

22.- MILLER W R, ROLLNICK S. Motivational interviewing: Preparing people to change addictive behavior. Nueva York: Guildford Press. 1991.

23.- PIÑEIRO B, MÍGUEZ M; BECOÑA E. La entrevista motivacional en el tratamiento del tabaquismo: una revisión. Adicciones 2010; 22 (4): 353-64.

24.- FUENTES-PILA J M, CALATAYUD P, LÓPEZ E, CASTAÑEDA B. La entrevista motivacional: llave del proceso de cambio en la dependencia nicotin-tabáquica. Trastornos Adictivos 2005; 7: 153-65.

25.- LAI DTC, CAHILL K, QIN Y, TANG J L. Motivational interviewing for smoking cessation. Cochrane Database of Systematic Reviews 2010, Issue 1. Art. No.: CD006936. DOI: 10.1002/14651858.CD006936.pub2.).

26.- SILAGY C, LANCASTER T, STEAD L, MANT D, FOWLER G. Nicotine replacement therapy for smoking cessation. Cochrane Database Syst Rev 2004; 3: CD000146.

27.- ANTHONISEN N R, CONNETT J E, KILEY J P, ALTOSE M D, BAILEY W C, BUIST A S., et al. Effects of smoking intervention and the use of an inhaled anticholinergic bronchodilator on the rate of decline of $\mathrm{FEV}_{1}$. The Lung Health Study. JAMA 1994; 272: 1497-505.

28.- TØNNESEN P, MIKKELSEN K, BREMANN L. Nurse-conducted smoking cessation in patients with COPD, using nicotine sublingual tablets and behavioral support. Chest 2006; 130: 314-6.

29.- WAGENA E J, ZEEGERS M P A, VAN SCHAYCK $\mathrm{C}$ P, WOUTERS E F M. Benefits and risks of phar- 
macological smoking cessation therapies in chronic obstructive pulmonary disease. Drugs Safety 2003; 26: 381-403.

30.- FAGERSTROM K O, SCHNEIDER N G, LUNELL E. Effectiveness of nicotine patch and nicotine gum as individual versus combined treatments for tobacco withdrawal symptoms. Psychopharmacology 1993; 111: 271-7.

31.- KORNITZER M, BOUTSEN M, DRAMAIX M, THIJS J, GUSTAVSSON G. Combined use of nicotine patch and gum in smoking cessation: a placebo controlled clinical trial. Prev Med 1995; 24: 41-7.

32.- JIMÉNEZ-RUIZ C, ULIBARRI M, BESADA N, GUERRERO A, GARCÍA A, CUADRADO A. Progressive reduction using nicotine gum as a prelude to quitting. Nicotine Tob Res 2009; 11: 847-50.

33.- WU P, WILSON K, DIMOULAS P, MILLS E J. Efectiveness of smoking cessation therapies: a systematic review and meta-analysis. BMC Public Health 2006; 6: 300-6.

34.- TASHKIN D P, KANNER R, BAILEY W, BUIST S, ANDERSON P, NIDES M A, et al. Smoking cessation in patients with chronic obstructive pulmonary disease: a double blind placebo-controlled, randomized trial. Lancet 2001; 357: 1571-5.

35.- VAN SCHAYCK C P, KAPER J, WAGENA E J, WOUTERS E F M, SEVERENS J L. The cost-effectiveness of antidepressants for smoking cessation in chronic obstructive pulmonary disease (COPD) patients. Addiction. 2009; 104: 2110-7.

36.- GONZÁLES D, RENNARD S I, NIDES M, ONCKEN C, AZOULAY S, BILLING C B, et al. Varenicline, an $\alpha 4 \beta 2$ nicotinic acetylcholine receptor partial agonist, vs sustained release bupropion and placebo for smoking cessation: an randomized controlled trial. JAMA 2006; 296: 47-55.

37.- JORENBY D E, HAYS J T, RIGOTTI N A, AZOULAY $\mathrm{S}$, WATSKY E J, WILLIAMS K E, et al. Efficacy of varenicline, an $\alpha 4 \alpha \beta 2$ nicotinic acetylcholine receptor partial agonist, $v s$ placebo or sustained-release bupropion for smoking cessation: a randomized controlled trial. JAMA 2006; 296: 56-63.

38.- GARZA D, MURPHY M, TSENG LJ RIORDAN HJ, CHATTERJEE A. A double blind randomized placebo controlled pilot study of neuropsychiatric adverse events in abstinent smokers trated with varenicline or placebo. Biol Psychiatry 2011; 69: 1075-82.

39.- GUNNELL D, IRVINE D, L WISE L, DAVIES C, MARTIN R M. Varenicline and suicidal behaviour: a cohort study based on data from the General Practice Research Database. BMJ 2009; 339: b3805.

40.- SINGH S, LOKE Y K, SPANGLER J G, FURBERG C D. Risk of serious adverse cardiovascular events associated with varenicline: a systematic review and meta-analysis. CMAJ 2011; 183: 1359-66.

41.- PROCHASKA J, HILTON J. Risk of cardiovascular serious adverse events associated with varenicline use for tobacco cessation: systematic review and meta-analysis. BMJ 2012; 344: e2856.

42.- TASHKIN D, RENNARD S, HAYS J T, MA W, LAWRENCE D, LEE T C. Effects of Varenicline on smoking cessation in mild-to-moderate COPD: A Randomized Controlled Trial. Chest 2011; 139: 591-9.

43.- TØNNESEN P, CARROZZI L, FAGERSTRÖM K O, GRATZIOU C, JIMÉNEZ-RUIZ C, NARDINIE S, et al. Smoking cessation in patients with respiratory diseases: a high priority, integral component of therapy Eur Respir J 2007; 29: 390-417.

44.- TASHKIN D, MURRAY R. Smoking cessation in chronic obstructive pulmonary disease. Respiratory Medicine 2009; 103: 963-74.

45.- STRASSMANN R, BAUSCH B, SPAAR A, KLEIJNEN J, BRAENDLI O, PUHAN M A. Smoking cessation interventions in COPD: a network metaanalysis of randomised trials Eur Respir J 2009; 34 : 634-40.
Correspondencia a:

Dr. Sergio Bello S.

Instituto Nacional del Tórax.

J. M. Infante 717. Santiago, Chile.

E-mail: sergio.bello@torax.cl 